\title{
Estimation and Analysis of the Deformation of the Cardiac Wall using Doppler Tissue Imaging.
}

\author{
Valérie Moreau ${ }^{1,2} \quad$ Laurent D. Cohen ${ }^{1} \quad$ Denis Pellerin $^{3}$ \\ ${ }^{1}$ CEREMADE Université Paris 9 Dauphine, 75775 Paris cedex 16, France \\ ${ }^{2}$ now with Epidaure project at INRIA Sophia Antipolis \\ ${ }^{3}$ Saint George's Hospital, London, UK. \\ E-mail: moreau, cohen@ceremade.dauphine.fr, dpellerin@sghms.ac.uk
}

\begin{abstract}
This paper presents different ways to use the Doppler Tissue Imaging (DTI) in order to determine deformation of the cardiac wall. As an extra information added to the ultrasound images, the DTI gives the velocity in the direction of the probe. We first show a way to track points along the cardiac wall in a M-Mode image $(1 D+t)$. This is based on energy minimization similar to a deformable grid. We then extend the ideas to finding the deformation field in a sequence of $2 D$ images $(2 D+t)$. This is based on energy minimization including spatio-temporal regularization.
\end{abstract}

\section{Introduction}

Doppler Tissue Imaging (DTI) is a recent non invasive ultrasound technique which allows to measure the velocity of intramyocardial wall motion. Results consists of a color overlay superimposed to the grey-scale conventional image (see Figure 1). At first the frame rate and the temporal resolution did not allow a precise study of the cardiac wall velocities over a whole cardiac cycle. That is why cardiologists need M-mode images (bottom of Figure 1). In practice, the cardiologist chooses a segment on the $2 \mathrm{D}$ image and the M-mode image shows its evolution through time. Each column of the image $I(t, z)$ corresponds to the segment at a given time $t$. Whereas a $2 \mathrm{D}$ sequence acquired with our VINGMED ultrasound system consists of about 100 images per second, an M-mode image consists of more than 500 frames per second.

This work follows the work performed by the authors of [1] about M-mode images. This work consists of tracking the cardiac wall with a variant of active contours. The contour $C(t)=(t, f(t))$ deforms to minimize

$$
E(f)=\int_{0}^{T} \omega_{1}\left|f^{\prime}\right|^{2}+\omega_{2}\left|f^{\prime \prime}\right|^{2}+P_{\text {edge }}(C)+P_{\text {velocity }}(C) \text {. }
$$

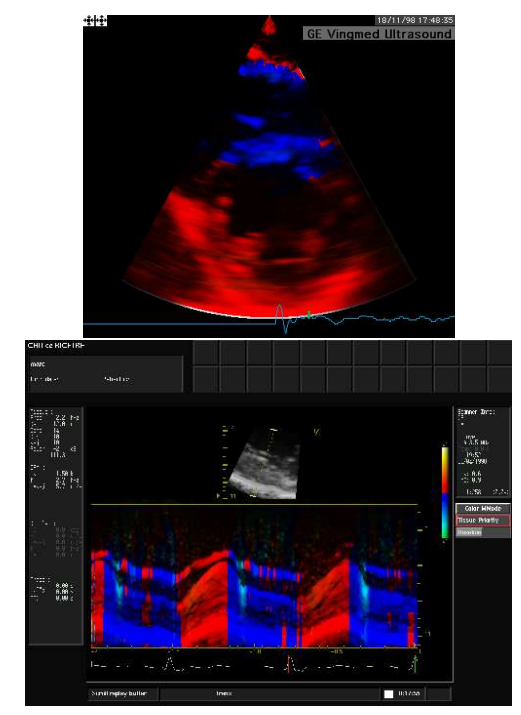

Figure 1. 2D and M-Mode DTI images.

$C(t)$ to the edge and $f^{\prime}(t)$ close to the velocity measured by the DTI. Authors of [1] have not only shown the advantage of using DTI in addition of the edge information but they have also used their results to show quantitatively that velocity of internal wall is higher than for external wall.

We will use different variational methods to study the deformation of the heart in M-Mode and 2D+t DTI images.

\section{Tracking through M-Mode Images}

As DTI provides quantitative information about intramyocardial wall motion, it helps to diagnose abnormal motion. But an M-mode image is rather hard to use because it provides many information and it is not easy to distinguish between close colors. An attractive way to present the results is to show tracking of different points of the cardiac wall using DTI. When we began this work, we used an automatic tracking which consisted of integration over time of 

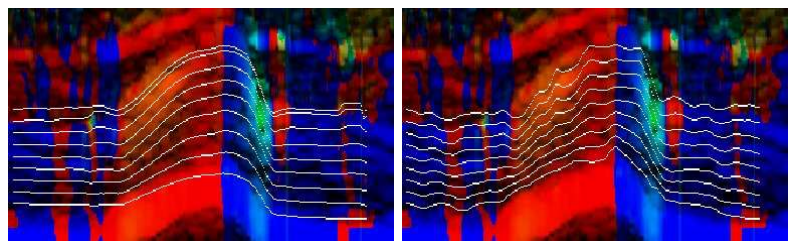

Figure 2. Initialization and result of the steepest gradient descent.

velocity measured by DTI, separately for each point (left of Figure 2). Since this tracking was not accurate enough, we improved the method by tracking simultaneously several points through the cardiac wall on the M-Mode image $I(t, z)$ making use of the velocity given by the DTI image $v_{D T I}(t, z)$. Points are chosen for the initial time $t=0$ along a hand given segment. They are regularly spaced on this vertical segment as seen on the left of images of figure 2. Let $\left\{C_{i}(t)=\left(t, y_{i}(t)\right)\right\}_{1 \leq i \leq N}$ be the curves which perform the tracking of each point. If these points are close enough, the curves $\left\{C_{1}, \ldots C_{N}\right\}$ must be consistent with one another. Indeed, all these points are in fact physical points of the same organ. In consequence, we will consider the set of curves $\left\{C_{1}, \ldots C_{N}\right\}$ as an elastic active net : $\left(t, y(t, s)=y_{s}(t)\right)_{s, t}$. Active nets or deformable grids are defined in $[3,4]$. The net deforms according to the minimization of an energy. It behaves as a set of active contours [2] that evolve together with mutual interaction. Our energy consists of three terms.

1. A regularization term as in [3, 4], $E_{\text {reg }}$ :

$\iint \alpha\left(\frac{\partial y}{\partial s}^{2}+\frac{\partial y^{2}}{\partial t}\right)+\beta\left(\frac{\partial^{2} y^{2}}{\partial s^{2}}+2{\frac{\partial^{2} y^{2}}{\partial s t}}^{2}+\frac{\partial^{2} y^{2}}{\partial t^{2}}\right)$

This term enables interaction between curves. The first derivatives make the net contract and the second derivatives enforce smoothness and rigidity. Coefficients $\alpha, \beta$ are positive. They were chosen experimentally according to the physical properties of the cardiac wall. Coefficient $\alpha$ should be small because the net should not contract too much.

2. An external term which attracts the derivative close to the given velocity measured by the DTI $E_{\text {velocity }}$ : $\iint\left(\frac{\partial y}{\partial t}-v_{D T I}(t, y(t, s))^{2}\right.$. This is an extension in two dimensions of [1].

3. The last term is also an external term, $E_{\text {grey }}$ : $\iint \sum_{k=-2}^{k=2}\left(I(t, y(t, s)+k)-I(0, y(0, s)+k)^{2}\right.$. As DTI is not sufficient, we want to keep the grey level nearly constant along each curve.

The net $y$ is obtained by the minimization of $E(y)=$ $E_{\text {reg }}(y)+\mu E_{\text {grey }}(y)+\lambda E_{\text {velocity }}(y), \mu$ and $\lambda$ are positive coefficients. As we know that grey level of a physical point is actually not quite constant through time in an ultrasound sequence, the third constraint must be very soft, and $\mu$ should be small compared to the other coefficients. We proceed as in $[1,5]$ and use a steepest gradient descent, discretization was done by finite differences. The energy can have many local minima. Therefore we must use an initialization close to the solution. We choose the previous algorithm which consists of the integration of the velocity measured by the DTI : $y(t+\Delta t, s) \approx y(t, s)+\Delta t v(t, y(t, s))$ for all $s$ (left of Figure 2). We finally obtain the result of right of figure 2 . We observe that the curves follow precisely the deformation of the cardiac wall compared to manual tracing of curves by the cardiologist. So our tracking algorithm is improved and has been of much help for cardiologists in order to study various use of DTI images $[6,7]$.

\section{Deformation Field in a 2D Image Sequence}

Our next interest is the velocity fields in 2D image sequences, which became possible since we got more powerful computers and better quality ultrasound images. Two difficulties arise from these 2D sequences: a low temporal resolution and the incomplete DTI information: the DTI only measures the velocity in the direction of the probe. Human and rat DTI sequences were provided by Drs Denis Pellerin and Colette Veyrat from Bicêtre Hospital. A DTI sequence consists of two types of data: the velocity $v_{D T I}(x, y, t)$ measured by the DTI in the direction of the probe $\left(x_{D T I}, y_{D T I}\right)$ and the conventional ultrasound sequence $I(x, y, t)$. We will use the second data to compensate the gaps of DTI.

The velocity measured by DTI provides a first linear constraint on the deformation field $(u, v): x_{D T I} u+y_{D T I} v=$ $v_{D T I}$. From the greyscale conventional ultrasound sequence, we can calculate the optical flow [8,9], that is the apparent motion. Optical flow is based on the hypothesis that the brightness of a point $I(x(t), y(t), t)$ is constant through time. Using the chain rule for differentiation we see that $\frac{\partial I}{\partial x} \frac{\partial x}{\partial t}+\frac{\partial I}{\partial y} \frac{\partial y}{\partial t}+\frac{\partial I}{\partial t}=0$. The optical flow equation becomes $I_{x} u+I_{y} v+I_{t}=0$, where $(u, v)=\left(\frac{\partial x}{\partial t}, \frac{\partial y}{\partial t}\right)$ is the unknown flow field. It does not define a unique solution. In fact, the optical flow gives the deformation in the direction of the gradient of the image. The method of Horn and Schunck ( [8] ) consists of including this constraint in an energy with a regularization term.

Despite the fact that the constant brightness hypothesis is not strictly observed in ultrasound images because of speckle, the regularization term added can overcome these problems if it is strong enough. For example, the authors of [10] have experimented optical flow techniques on synthesized ultrasound sequence and demonstrated the validity of using Horn and Schunck methods.

In our case, we also have with DTI a second linear constraint on the deformation field. But these two linear constraints are not always linearly independent. So the solu- 
tion cannot be the resolution of the system. Since moreover ultrasound images are very noisy, we propose a solution inspired by Horn and Schunck method. The field $(u(x, y, t), v(x, y, t))$ we are looking for will satisfy, when possible, the optical flow constraint, the agreement with the DTI velocity and a first order regularity constraint. We include these three constraints in an energy minimization. For each frame $t$ :

$$
\begin{aligned}
& E(u, v)=\iint_{\Omega} \alpha\left(x_{D T I} u+y_{D T I} v-v_{D T I}\right)^{2} \\
& \quad+\alpha\left(I_{x} u+I_{y} v+I_{t}\right)^{2}+\beta\left(\|\nabla u\|^{2}+\|\nabla v\|^{2}\right) .
\end{aligned}
$$

where $\Omega$ is the image domain. Coefficients $\alpha$ and $\beta$ are positive and are chosen experimentally. We minimize this energy with a steepest gradient descent.

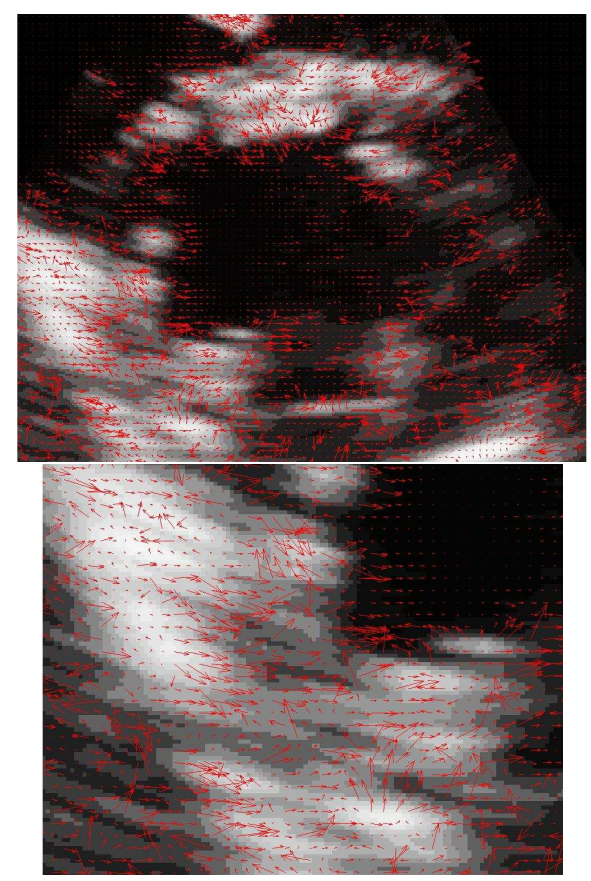

Figure 3. Velocity field with our method.

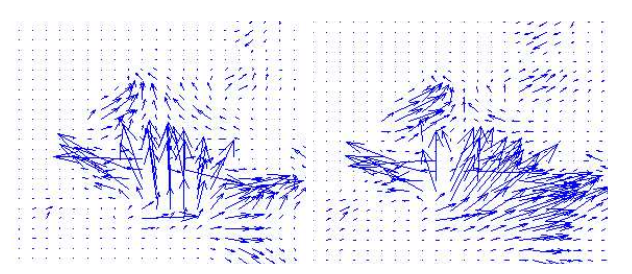

Figure 4. Improvement provided by DTI. Optical Flow without and with DTI

An example of result is given in Figure 3. The results are satisfying compared with simple optical flow as shown in figure 4. Direction is modified and we get a more regular field without increasing the diffusion. But some problems due to the acquisition persist. First, gaps in the lateral wall imply gaps in optical flow for which DTI cannot always compensate since it is always in the direction of the probe. Second, the image of lateral walls consists of horizontal spots because the transmitted signal is oblique to them, and so the gradient of the image is not really what expected, and so is optical flow. However, the deformation field is quite accurate and regular around the anterior and posterior walls.

In order to fill gaps, we adapt the ideas developped in [9] and include a temporal regularization. This asssumption of temporal regularity is relevant since the temporal resolution is high enough to provide small displacement between successive images. The minimized energy is then :

$$
\begin{aligned}
& E(u, v)=\iint_{\Omega \times[0, T]}\left(x_{D T I} u+y_{D T I} v-v_{D T I}\right)^{2} \\
& +\alpha\left(I_{x} u+I_{y} v+I_{t}\right)^{2}+\beta\left(\|\nabla u\|^{2}+\|\nabla v\|^{2}\right) .
\end{aligned}
$$

Here $\nabla$ is a $3 D$ gradient. It ensures interaction between different frames. An example of result is shown in figure 5. As in [9], the result is much more coherent. We can see that missing information is completed by the neighbouring images. The velocity field is much more regular and complete around the myocardium wall than the first one.

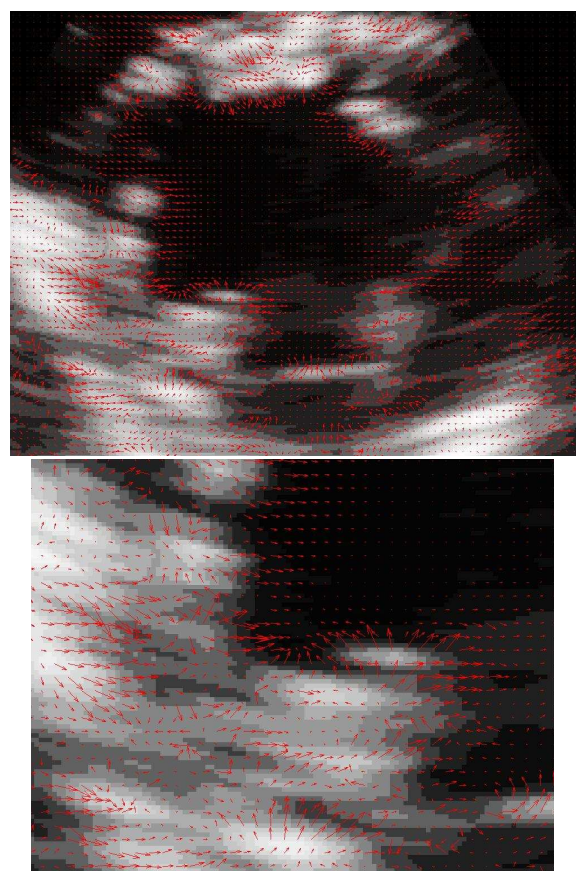

\section{Figure 5. Velocity field with a spatio-temporal regularization.}

If the displacement field is still not satisfying, we have to use a fast algorithm to improve ultrasound images by enhancing edges. We chose the "sticks algorithm" introduced by [11] to reduce speckle noise and improve the edge information as shown in figure 6 . We must carefully select the 


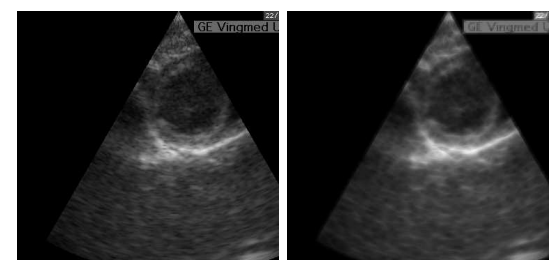

Figure 6. The sticks algorithm. Original Image and Enhanced Image.

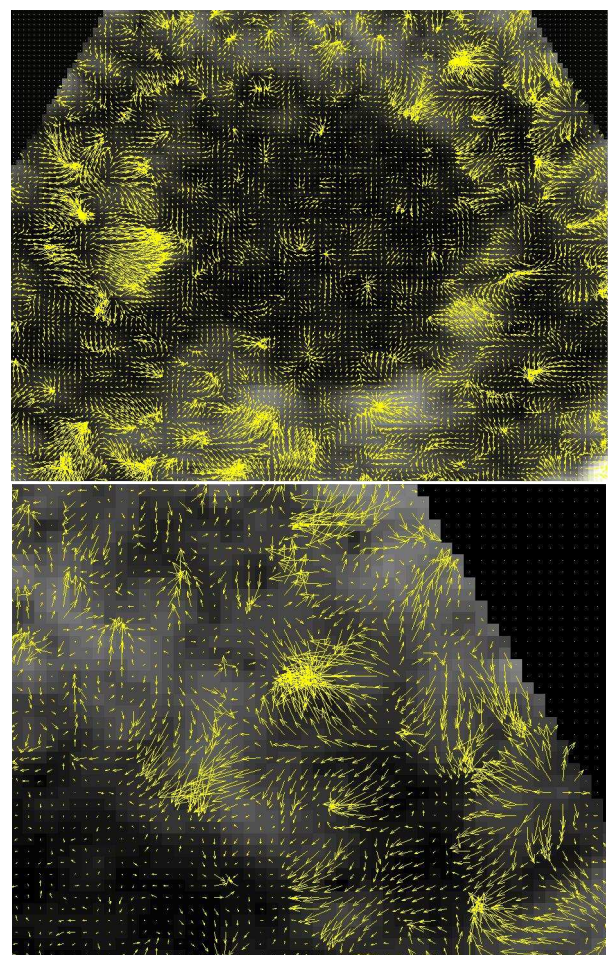

Figure 7. Velocity field processed.

regularization so that the image is still textured for the optical flow to be efficient. We then use the enhanced image instead of the original ultrasound image in the algorithms above. Figures 7 and 8 show the improvement of this preprocessing. We see that the impact of the speckle noise on the velocity field is decreased.

We checked all these results by deforming an image with the velocity field and comparing to the following image. In every case we noticed that the deformed image is close to the following one both visually and based on mean square error. But an important challenge is a real validation of this velocity field.

\section{Conclusion}

We have presented different ways to use the Doppler Tissue Imaging (DTI) in order to determine deformation of the cardiac wall. We first showed a way to track points along the cardiac wall in a M-Mode image $(1 \mathrm{D}+\mathrm{t})$, based on a de-

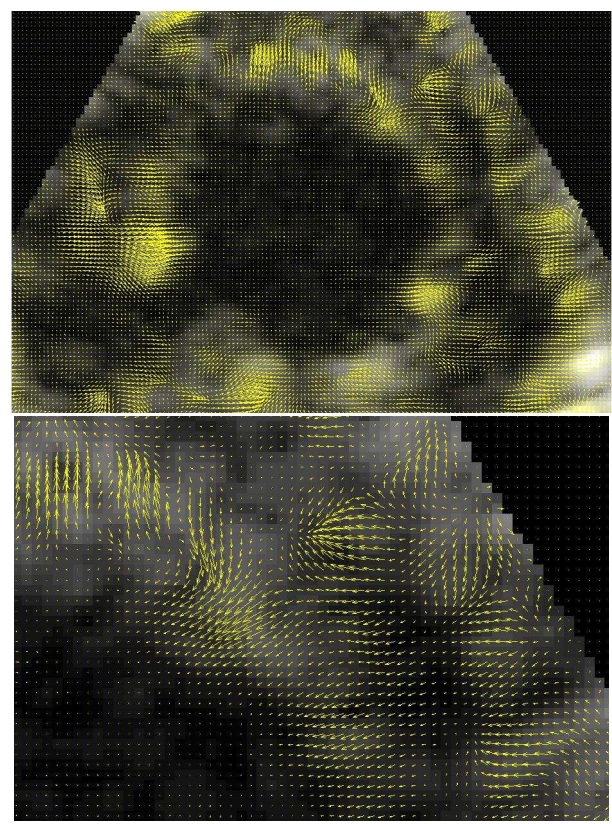

\section{Figure 8. Velocity field processed on the same sequence after enhancement.}

formable grid energy minimization. We then showed how to estimate the deformation field in a sequence of 2D images, based on energy minimization including spatio-temporal regularization and a priori constraints. Future work includes validation and analysis of the velocity field.

\section{References}

[1] Laurent D. Cohen, F. Pajany, D. Pellerin, and C. Veyrat. Cardiac wall tracking using doppler tissue imaging (DTI). In In Proc. ICIP'96, Lausanne, Switzerland, Sept. 1996.

[2] M. Kass, A. Witkin and D. Terzopoulos. Snakes: Active Contour Models. IJCV 1(4), 1988.

[3] M. Bro-Nielsen. Active Nets and Cubes. Technical Report, Technical University of Denmark, Nov. 1994.

[4] K. Yoshino, T. Kawashima and Y. Aoki. Dynamic Reconfi guration of Active Net Structure. Asian Conference on Computer Vision, Nov. 1993.

[5] L.D. Cohen. On active contours models and balloons. CVGIP:IU, 53(2), March 1991.

[6] Veyrat C, Pellerin D, Cohen L, Larrazet F, Extramiana F, and Witchitz S. Spectral, one-or two-dimensional tissue velocity doppler imaging: which to choose? Cardiology, 9(1), 2000.

[7] Pellerin D, Berdeaux A, Cohen L, Giudicelli JF, Witchitz S. and Veyrat C. Comparison of two myocardial velocity gradient assessment methods during dobutamine infusion using doppler myocardial imaging. Journal of the American Society of Echocardiography, 12, 1999.

[8] B.K.P. Horn and B.G. Schunck. Determining Optical Flow. Artifi cial Intelligence, (17), 1981

[9] J. Weickert and C. Schnörr. Variational Optic Flow Computation with a Spatio-Temporal Smoothness Constraint. Journal of Mathematical Imaging and Vision, 14(3), May 2001.

[10] P. Baraldi, A. Sarti, C. Lamberti, A. Prandini, F. Sgallari. Evaluation of Differential Optical Flow Techniques on Synthesized Echo Images. IEEE Transactions on Biomedical Engeneering, 43(3), March 1996.

[11] R.N. Czerwinski, D.L. Jones and W.D. O'Brien. Detection of Lines and Boundaries in Speckle Images. Application to Medical Ultrasound. IEEE TMI, Vol 18(2), Feb. 1999. 